\title{
Polarimetric Optical High-Voltage Sensor Using Synthetic-Heterodyne Demodulation and Hilbert Transform With Gain Control Feedback
}

\author{
José Henrique Galeti, Student Member, IEEE, Ricardo Tokio Higuti, Member, IEEE, Cláudio Kitano, \\ and Michael J. Connelly, Senior Member, IEEE
}

\begin{abstract}
Optical voltage sensors are widely used for highvoltage (HV) sensing, but the measurement accuracy is often compromised by a sensitivity drift due to variations in the ambient conditions. Synthetic-heterodyne demodulation is a useful technique for dynamic displacement measurements using interferometric sensors as it can provide a detected signal that is immune to an interferometric drift. In this paper, a new synthetic-heterodyne technique, employing the Hilbert transform and gain control feedback, is applied to a polarimetric-based optical sensor system and used to measure an $\mathrm{HV}$ signal. The system comprises an $\mathrm{He}-\mathrm{Ne}$ laser source, optical phase modulator, and HV sensor located between two polarizers followed by a photodetector and real-time electrical signal acquisition and processing. The use of a control loop results in significant improvements in the stability and accuracy of the detected $\mathrm{HV}$ signal. The sensor system was used to measure a $60-\mathrm{Hz} \mathrm{HV}$ signal with an amplitude range of $300 \mathrm{~V}$ to $7.5 \mathrm{kV}$. The sensor was also used to successfully measure the total power line harmonic distortion of a highly distorted $4 \mathrm{kV}$ signal. The experimental results show very good agreement with measurements obtained by using the industry standard signal coincidence method.
\end{abstract}

Index Terms-High-voltage sensor, instrumentation, measurement, interferometry, metrology, phase measurement.

\section{INTRODUCTION}

$\mathbf{N}$ OWADAYS, bulky iron core and capacitor voltage transformers are continuously under review in modern power systems due to their higher cost, safety implications and surrounding plant due to failure, long setup time and substation land requirement. In 2011 [1] presented an experimental comparison between the performances of a conventional magnetic Voltage Transformer (VT) sensor for measuring High-Voltage (HV) and an Optical Voltage Sensor (OVS) based on the Pock-

Manuscript received May 27, 2016; revised September 7, 2016; accepted October 9, 2016. Date of publication October 11, 2016; date of current version November 22, 2016. This work was supported in part by the CAPES Foundation, Ministry of Education of Brazil (7730-14-4), in part by the CNPq National Council of Research and Development, Brazil (478817/2012-6), and in part by the Enterprise Ireland (CFTD/07/IT/312b).

J. H. Galeti, R. T. Higuti, and C. Kitano are with the Department of Electrical Engineering, São Paulo State University, Ilha Solteira, São Paulo 15385-000, Brazil (e-mail: jhgaleti@gmail.com; higuti@dee.feis.unesp.br; kitano@dee.feis.unesp.br).

M. J. Connelly is with the Optical Communications Research Group, Department of Electronic and Computer Engineering, University of Limerick, Limerick V94 T9PX, Ireland (e-mail: michael.connelly@ul.ie).

Color versions of one or more of the figures in this paper are available online at http://ieeexplore.ieee.org

Digital Object Identifier 10.1109/JSTQE.2016.2616647 els effect. As a result, it was found that the OVS offers better accuracy, higher dynamic range and bandwidth, has superior transient response and may respond better for switching surge measurements. Moreover, the saturation of the magnetic susceptibility of the magnetic VT iron core limits the linear response range, and the thermal heating caused by surge currents can lead to explosions. The large amount of oil that is used for insulation, as well as the parts made of copper, ceramic and iron lead to large volume and weight [2]. The use of optical fibers to carry the measurement light signal to and from the sensor-head also provides the ability to control power systems remotely, electrically isolating the observer from the HV environment, thereby adding immunity against lightning and electromagnetic interference. In addition, since digital control and protection systems have been introduced in electric power systems, reduction of the amount of power consumption during measurement of conventional voltage transformers, as well as the mitigation of electromagnetic interference, are required. Consequently, OVSs are safer as they provide dielectric insulation and are advantageous because they are smaller and lighter than magnetic VTs. Finally, the output signals from OVSs are compatible with the modern smart grid and electric power industry.

OVSs for HV AC measurements have been widely studied since the 1990s; most of them employ bulk Pockels crystals as the sensing element inside a polarimetric layout. For these types of Pockels effect based OVSs, electro-optic crystals, such as Lithium Niobate $\left(\mathrm{LiNbO}_{3}\right)$, Lithium Tantalite $\left(\mathrm{LiTaO}_{3}\right)$ or Bismuth Germinate (BGO), are preferred. BGO is usually chosen as the sensing element because it possesses neither natural birefringence with temperature dependence or optical activity [3]. On the other hand, $\mathrm{LiNbO}_{3}$ and $\mathrm{LiTaO}_{3}$ crystals are more attractive for integrated optical OVSs [2], [4]. Although a large number of different experimental arrangements and operating principles have been published, most OVSs employ classical mathematical tools such as the Jones matrix [5], [6] or adaptations of classical techniques usually applied to interferometers, such as optical phase detection schemes applied to optical fiber gyroscopes [7]. Furthermore, various configurations based on fiber Bragg gratings mounted on piezoelectric transducers have been developed to measure HV [8], [9]. However, these methods have limited measurement ranges [10].

In this work we explore the Pockels effect in $\mathrm{LiNbO}_{3}$. In such schemes, an electro-optic crystal is employed, where an exter- 
nal electric field is applied by parallel electrodes to a convenient direction along some of the crystal axes. A linearly polarized light source is input to the crystal by the use of a polarizer whose transmission axis is at an angle of $45^{\circ}$ to one of its principal axis, which couples both the ordinary and extraordinary polarization modes of the birefringent crystal. The two polarization states are the equivalents of the signal and reference beams of an interferometer. The relative phase retardation between the polarization states is a function of the voltage applied to the Pockels cell. As orthogonal states of polarization do not interfere, a polarization analyzer is used to resolve the two states into a common azimuth so that they interfere to produce a detected output intensity given by $v(t)=A(1-V \cos \Gamma) / 2$, where $\Gamma=\varphi(t)+\varphi_{0}$ is the phase retardation, $A$ includes the laser power and photodetector responsivity, $\varphi_{0}$ is the quasi-static phase difference between the ordinary and extraordinary rays in the Pockels cell and $\varphi(t)$ is the phase shift induced by the applied voltage [11]. The modulator is usually biased with a fixed $\pi / 2$ phase delay (quadrature operation), which can be achieved by using a $\lambda / 4$ waveplate in series with the cell. As can be seen, there is a similarity between the form of $v(t)$ for the electro-optic intensity modulator and that for a two beam interferometer, except for the algebraic sign (-) and that the visibility $V$ is equal to unity in the electro-optic system. Consequently, most phase detection techniques for an interferometer can also be applied to the OVS. However, in practice small alignment errors can occur between the direction of beam propagation or its polarization relative to the crystal axes, and thus, $V$ becomes smaller than unity, as occurs in practical interferometers. When a given interferometer demodulation method is independent of $\varphi_{0}$ and thereby fluctuations due for example to temperature drift, there is no need to operate at quadrature and so a quarter wave-plate is not necessary thereby simplifying the system.

However, most of detection methods described above employ open loop schemes, making them susceptible to the signal fading effect caused by system temperature drifts; consequently it is difficult to improve the accuracy of these $\mathrm{LiNbO}_{3} \mathrm{OVSs}$. Recently, an interesting OVS based on an optical fiber Michelson interferometer, that is insensitive to the intensity fluctuations of laser and using the Phase Generated Carrier (PGC) method was demonstrated [10]. This method also eliminates signal fading caused by random fluctuations of temperature or pressure at low frequencies. However, this technique, while similar to the method presented here, is an open loop scheme that is based on utilizing the fundamental and second harmonic of the Bessel function based Fourier expansion of the received signal followed by an arctan algorithm, and so required a phase unwrapping procedure, which complicates the signal processing.

We previously demonstrated a closed loop optical phase demodulation scheme, which was applied effectively to detect microscopic displacement in piezoelectric actuators, by using a Michelson interferometer and synthetic-heterodyne technique [12]. This technique could also be applied to the OVS problem by improving the measurement process, making it immune to the fading effect. However, that technique requires four synchronized local oscillators, which needs specific controls. Furthermore, the use of spectral components around the fundamental and double frequencies of a carrier wave, even when optimized,

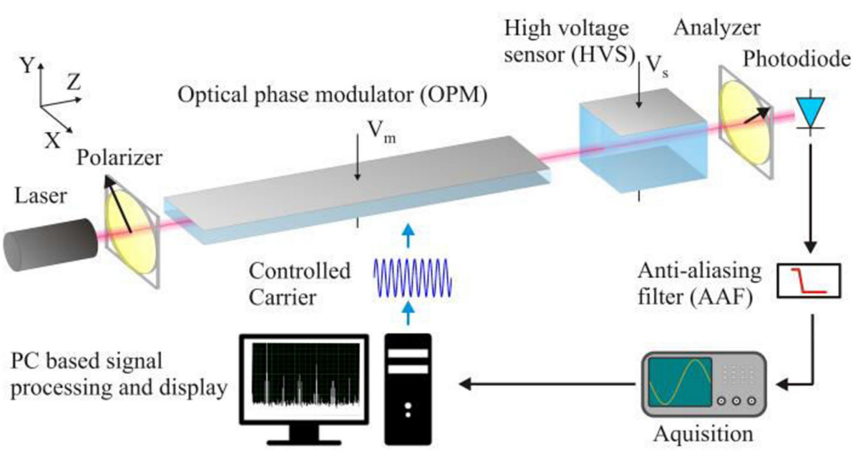

Fig. 1. Experimental setup.

achieve the same power level as the signal, and hence generates a Signal-to-Noise Ratio (SNR) lower than that achievable in this work. The new technique improves the performance of the method, eliminating the need for local oscillators by utilizing the Hilbert transform and using only the components without the signal carrier, which expands the dynamic range for a given acquisition frequency leading to an improved SNR.

Section II gives a brief description of the theory and system implementation. Section III describes the study of gain feedback control and Section IV presents experimental results followed in Section $\mathrm{V}$ by conclusions.

\section{THEORY AND IMPLEMENTATION}

A schematic diagram of the sensor system is shown in Fig. 1, illustrating the $\mathrm{X}, \mathrm{Y}$ and $\mathrm{Z}$ coordinates relative to the crystal axes. The optical source is a $632.8 \mathrm{~nm}$ He-Ne laser with an output power of $20 \mathrm{~mW}$. The laser beam was polarized at an angle of $45^{\circ}$ to the Y-axis, passed through the Optical Phase Modulator (OPM) and the High Voltage Sensor (HVS) and analyzed with a polarizer at an angle of $-45^{\circ}$ to the Y-axis. The resultant light beams are detected by an amplified PIN photodiode, passed through an Anti-Aliasing Filter (AAF) before acquisition using a digital oscilloscope and processed in realtime using LabVIEW. A sinusoidal carrier wave at frequency $f_{0}=12 \mathrm{kHz}$ and adjustable amplitude $V_{m}$ was applied to the OPM using a LABVIEW controlled function generator.

The OPM and HPV are Lithium Niobate crystals aligned in the Y-direction (natural birefringence) and Z-direction (nonnatural birefringence) respectively, with corresponding $\pi$ phase shift voltages $V_{\pi m}=(Y / Z) \lambda /\left(n_{e}^{3} r_{33}-n_{o}^{3} r_{13}\right)$ and $V_{\pi s}=$ $(Y / Z) \lambda / 2 n_{o}^{3} r_{22}$, where $\lambda$ is the optical wavelength, $n_{o}^{3}$ the ordinary refractive index, $n_{e}^{3}$ the extraordinary refractive index and the crystal electro-optic coefficients in respective directions are $r_{n m}$ [11]. The dimensions of the OPM and HVS dimensions were chosen to obtain a desired $V_{\pi m}=64.9 \mathrm{~V}$ and $V_{\pi s}=$ $3.77 \mathrm{kV}$. In the case of the OPM the $\mathrm{X}, \mathrm{Y}$ and $\mathrm{Z}$ dimensions are $10,1.1$ and $50 \mathrm{~mm}$ respectively for the HVS the $\mathrm{X}, \mathrm{Y}$ and $\mathrm{Z}$ dimensions are 20,9.92 and $10.26 \mathrm{~mm}$ respectively.

The detected voltage signal at the oscilloscope input is given by [12],

$$
v(t)=A\left\{1-V \cos \left[\Phi(t)+\Phi_{0}+\varphi(t)+\phi(t)\right]\right\}
$$

where $A$ is the average voltage, $V$ the visibility, $\Phi(t)$ and $\Phi_{0}$ the dynamic and static phase shifts induced by the OPM, $\varphi(t)$ 
is the phase induced by the HVS and $\phi(t)$ is a slowly varying phase shift caused by environmental drift.

Considering the voltage $V_{m}(t)=\left(V_{\pi m} C /\right) \cos \left(2 \pi f_{0} t\right)$ applied to the OPM, then

$$
\Phi(t)=C \cos \left(2 \pi f_{0} t\right)
$$

where $f_{0}$ and $C$ are the carrier frequency and the controlled modulation index applied to the OPM. The phase induced by the HVS is

$$
\varphi(t)=\pi \frac{V_{s}(t)}{V_{\pi s}}
$$

where $V_{s}(t)$ is the voltage applied to the HVS, whose magnitude is to be measured. By substituting (2) into (1) and defining

$$
\theta(t)=\Phi_{0}+\varphi(t)+\phi(t)
$$

(1) can be expanded into a DC term plus an infinite series of carriers located at integer multiples of $f_{0}$, multiplied by either $\sin \theta(t)$ or $\cos \theta(t)$,

$$
\begin{aligned}
v(t)= & A+A V J_{o}(C) \cos \theta(t)-2 A V \sum_{k=0}^{\infty}(-1)^{k} J_{2 k+1}(C) \\
& \times \cos \left[2 \pi(2 k+1) f_{0} t\right] \sin \theta(t) \\
& +2 A V \sum_{k=1}^{\infty}(-1)^{k} J_{2 k}(C) \cos \left(4 \pi k f_{0} t\right) \cos \theta(t)
\end{aligned}
$$

where $J_{i}(C)$ are Bessel functions of the first kind of order $i$ with argument $\mathrm{C}$. A typical measured signal and its spectrum are shown in Fig. 2, where the sampling points are joined by straight-line segments. The signal is very similar to the form of (1) for a sinusoidal $V_{s}(t)$.

In the synthetic-heterodyne demodulation scheme used in our previous work [12], two terms in (5) with $\sin \theta(t)$ and $\cos \theta(t)$ are processed to obtain a signal proportional to $\theta(t)$. When the terms at $f_{0}$ and $2 f_{0}$ are used the maximum detectable measurand signal bandwidth is $f_{0} / 2$. In this case the minimum received signal bandwidth, to allow successful measurand signal demodulation is $2.5 f_{0}$, which by the Nyquist sampling theorem requires a minimum sampling rate of $5 f_{0}$, i.e. 10 times the bandwidth of the measured signal.

The method proposed here only requires six times the sample rate, by using the terms centered at $f_{0}$ and the $J_{o}(C)$ term centered at DC in (5). Subtracting the average $A$ from (5) and applying a lowpass filter with (bandwidth of $5 \mathrm{kHz}$ ) gives

$$
S_{1}(t)=A V J_{o}(C) \cos \theta(t)
$$

Also (5) bandpass filtered around $f_{0}$ (bandwidth from $7 \mathrm{kHz}$ to $12 \mathrm{kHz}$ ) and divided by 2 , gives

$$
S_{h}(t)=-A V J_{1}(C) \cos \left(2 \pi f_{0} t\right) \sin \theta(t)
$$

It is desirable to obtain, from (7), a signal without the term $\cos \left(2 \pi f_{0} t\right)$. In our previous work [12] this was achieved by multiplying (7) by a local oscillator and lowpass filtering, however, a more complex method to control the local oscillator gain
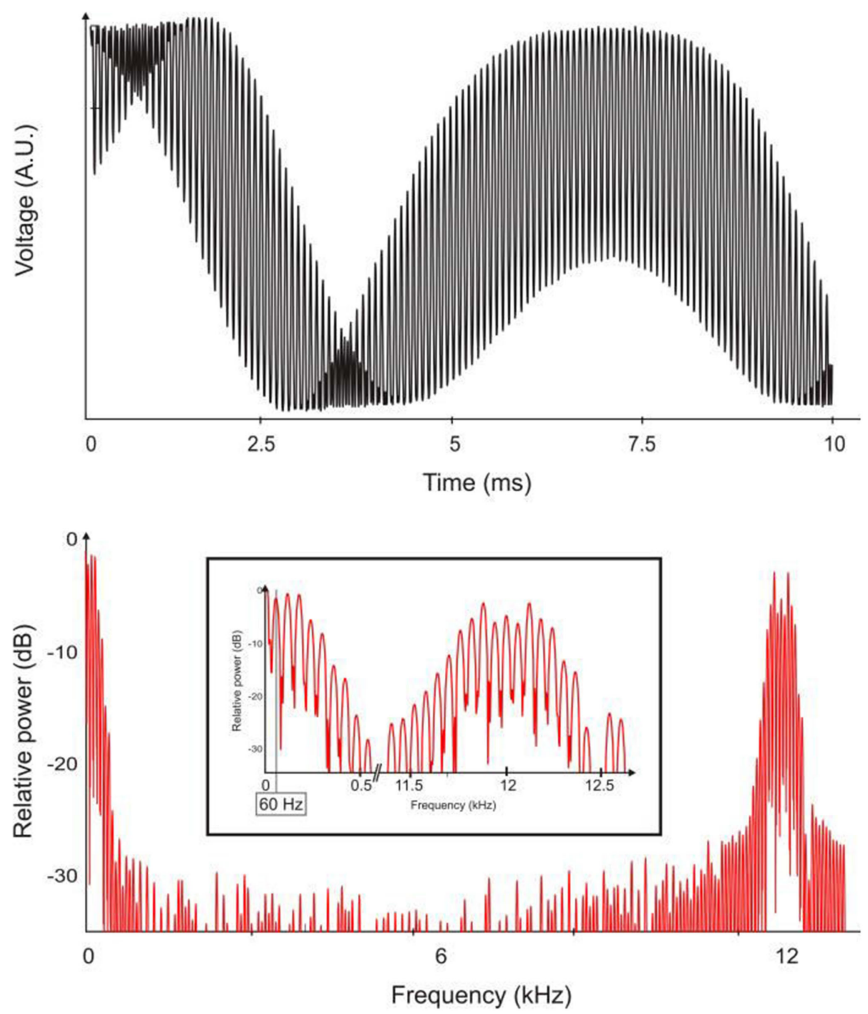

Fig. 2. (Top) typical measured signal and (bottom) its spectrum. The inserted detail is the spectrum at low frequencies showing the terms centered at the $12 \mathrm{kHz}$ carrier frequency and the measured $60 \mathrm{~Hz}$ signal, $V_{s}(t)$.

and phase was necessary. In this work, to simplify the method, we use apply instead a Hilbert transform to obtain the analytical signal of (7), given by

$$
\begin{aligned}
E n v & =\left|A V J_{1}(C) \sin \theta(t)\right| \\
P h & =2 \pi f_{0} t
\end{aligned}
$$

where $E n v$ and $P h$ are the analytical signal envelope and instantaneous phase respectively $[13,14]$. (8) cannot be used to obtain the sign of $\sin \theta(t)$; in practice it can be determined using (9). When $\sin \theta(t)$ is negative (9) becomes $P h=2 \pi f_{0} t-\pi$, so by subtracting $2 \pi f_{0} t$ from (9) the result is either $-\pi$ or 0 from which the sign of $\sin \theta(t)$ can be determined. Once the sign of $\sin \theta(t)$ is known and using (8) we get

$$
S_{2}(t)=A V J_{1}(C) \sin \theta(t)
$$

assuming $A V J_{1}(C)>0$. From (6) and (10) and defining $S_{3}=$ $S_{1} d S_{2} / d t-S_{2} d S_{1} / d t$ we get

$$
S_{3}(t)=(A V)^{2} J_{0}(C) J_{1}(C) \dot{\theta}(t)
$$

where $\dot{\theta}(t)$ is the time derivative of $\theta(t)$. If the lowpass filters are ideal the sensor bandwidth is $f_{o} / 2$, i.e. $6 \mathrm{kHz}$. In order to obtain $\dot{\theta}(t)$, the factor $(A V)^{2} J_{0}(C) J_{1}(C)$ must be known. In conventional synthetic-heterodyne demodulation, this requires direct measurement of $A V$ and $C$, which particularly in the latter case can be difficult, although there are some reliable techniques 


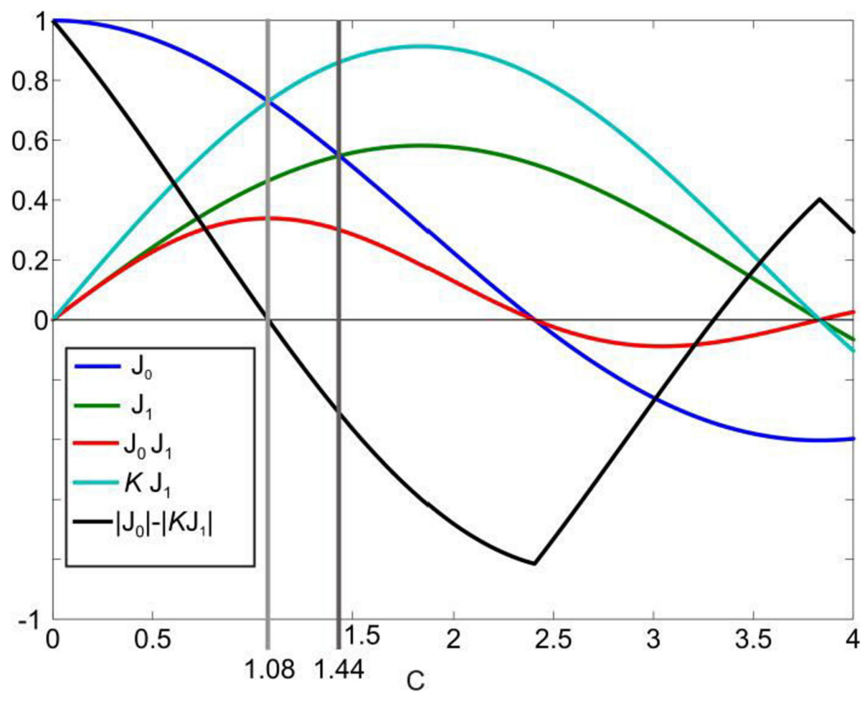

Fig. 3. Bessel functions and combinations.

available to obtain the value of $(A V)^{2} J_{0}(C) J_{1}(C)$ [15]. In a less mechanically stable setup or where the received optical power and visibility can vary significantly with time, it is desirable to obtain a signal proportional to $\dot{\theta}(t)$ that is independent of $A V$. Defining $S_{v}=S_{3} /\left(S_{1}^{2}+S_{2}^{2}\right)$ gives

$$
S_{v}(t)=\frac{J_{0}(C) J_{1}(C) \dot{\theta}(t)}{J_{0}^{2}(C) \cos ^{2} \theta(t)+J_{1}^{2}(C) \sin ^{2} \theta(t)}
$$

If $C$ is chosen such that $J_{0}(C)=J_{1}(C)$, then the demodulated output signal obtained from (12), becomes

$$
S_{v}(t)=\dot{\theta}(t)
$$

which is self-calibrated and independent of ambient fluctuations and $A V$ thereby improving measurement stability and accuracy. Considering $\dot{\varphi}(t)>\dot{\phi}(t)$ and $\dot{\varphi}(t)>\dot{\Phi}_{0}, V_{s}$ is obtained by highpass filtering and integrating $S_{v}$ starting at some reference (arbitrary) time $t_{0}$, and substituting into (3) to get

$$
V_{s}(t)=\frac{V_{\pi s}}{\pi} \int_{t_{0}}^{t} \dot{\varphi}(t)
$$

The above analysis assumes that $J_{0}(C)=J_{1}(C)$. In our previous work the condition $J_{1}(C)=J_{2}(C)$ was used. The aim to obtain a self-calibration method (i.e. $\theta(t)$ independent of $A V$ ) was obtained, initially, by measuring $C$ and self-correcting its value to $C=4.44$ at which $\left|J_{1}(C) J_{2}(C)\right|$ is at its maximum value of 0.051 , for $C>\pi$ (which was a necessary measurement condition) [16]. To improve the SNR by increasing $\left|J_{1}(C) J_{2}(C)\right|$, a feedback control loop technique was used [12] to set $C$ to a value at which $\left|J_{1}(C) J_{2}(C)\right|$ was equal to 0.21 , which is very close to its maximum possible value of 0.22 . In this work, which uses the $J_{0}(C)$ and $J_{1}(C)$ terms in (5), the maximum value of $\left|J_{0}(C) J_{1}(C)\right|$ can be increased to 0.34 by setting $C=1.08$ as shown in Fig. 3, which leads to a significant improvement in the SNR. Unfortunately the necessary condition, $J_{0}(C)=J_{1}(C)$ occurs when $C=1.44$; how-

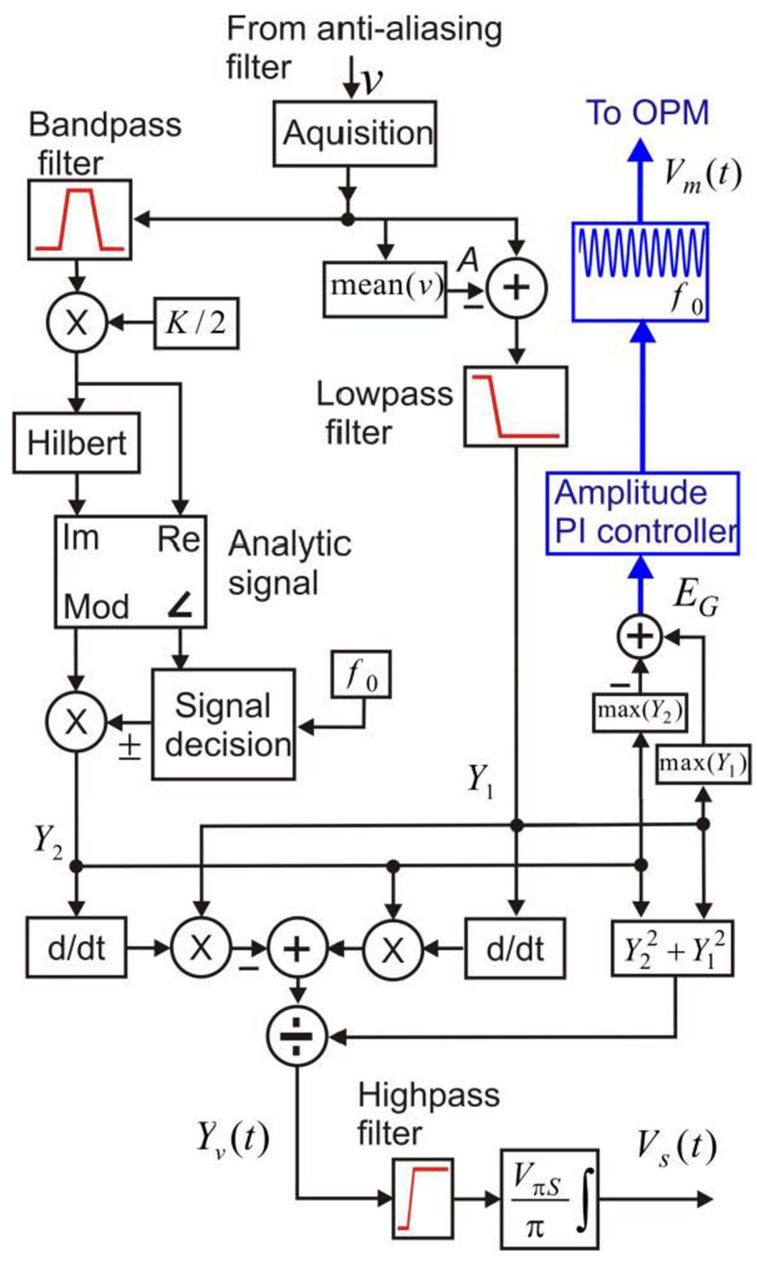

Fig. 4. Signal processing and control loop schematic diagram.

ever this condition can be replaced by a new necessary condition $J_{0}(C)=K J_{1}(C)$, where $K$ is a constant multiplied by (7). The $K$ constant is calculated $\left[K=J_{0}(C) / J_{1}(C)\right]$ as $\pi / 2$, considering that $C=1.08$ is the value required to maximize the product $\left|J_{0}(C) J_{1}(C)\right|$.

Even though it is possible to manually adjust the value of $C$, it is appropriate to control it using an error signal such as $J_{0}(C)-K J_{1}(C)$ (Fig. 3). A feedback control loop, in addition to setting $C$ to an optimum value, is also able to compensate for variations in $V_{\pi m}$, due mainly to temperature and the AAF frequency response as described in the next section.

\section{GAIN FEEDBACK CONTROL}

The IEC 61850 standards, used for instrument transformers in $\mathrm{HV}$ power quality measurements, requires a prior defined accuracy (class dependent) of between $80 \%$ and $120 \%$ of the nominal voltage [17].

As schematically shown in Fig. 4, the signal processing algorithm and control loop was designed to have the activated control around $V_{s}(t)$ with a nominal voltage $V_{n}$. The minimum controlled $V_{s}(t)$ is obtained by the HVS with $V_{\pi s} \leq 0.8 V_{n}$ leading to $\varphi(t) \geq \pi$ at (3), with is a sufficient condition to 
automatically determine $A$ and control the value of $C$. Whenever $V_{s}(t)$ is less than $V_{\pi s}(\varphi(t)<\pi)$ the feedback loop is disabled, nevertheless the system remains working using previously stored $A$ and $C$ values; in this sense, it has a higher expected accuracy whenever $V_{s}(t)>V_{\pi s}$.

If the AAF frequency response is considered, the correct form of (6) and (10) can be expressed as (15) and (16), where $G_{0}$ and $G_{1}$ are the DC and $f_{0}$ AAF voltage gains respectively. $K$ is the constant used to obtain maximum value of $\left|J_{0}(C) J_{1}(C)\right|$ product, as a consequence improves the SNR, as described in Section II,

$$
\begin{aligned}
& Y_{1}(t)=A V G_{0} J_{0}(C) \cos \theta(t) \\
& Y_{2}(t)=A V K G_{1} J_{1}(C) \sin \theta(t)
\end{aligned}
$$

In the same manner as (12) calculating $Y_{v}=\left(Y_{1} \dot{Y}_{2}-\right.$ $\left.Y_{2} \dot{Y}_{1}\right) /\left(Y_{1}^{2}+Y_{2}^{2}\right)$ gives

$$
Y_{v}(t)=\frac{K G_{0} G_{1} J_{0}(C) J_{1}(C) \dot{\theta}(t)}{G_{0}^{2} J_{0}^{2}(C) \cos ^{2} \theta(t)+K G_{1}^{2} J_{1}^{2}(C) \cos ^{2} \theta(t)}
$$

By setting $C$ to an appropriate value, (17) will be equal to $\dot{\theta}(t)$. This can be achieved by suitable control of the $V_{m}(t)$ amplitude using a feedback loop. $Y_{1}(t)$ and $Y_{2}(t)$ will reach respective peak values of $A V G_{0} J_{0}(C)$ and $A V K G_{1} J_{1}(C)$, when in (4) $\varphi(t) \geq \pi$ gives $\theta(t) \geq \pi$ and assuming that $\dot{\varphi}(t)>\dot{\phi}(t)$ and $\dot{\varphi}(t)>\dot{\Phi}_{0}$. Defining a gain error signal

$$
E_{G}=A V\left[G_{0} J_{0}(C)-K G_{1} J_{1}(C)\right]
$$

which can be obtained by measuring the peak values of $Y_{1}(t)$ and $Y_{2}(t)$ over a time span equal to or exceeding the period of $\varphi(t)$; furthermore, through $V_{m}(t)$ amplitude $\left(V_{\pi m} C / \pi\right)$ the $C$ value can be controlled. A PI control loop was used to control the amplitude of $V_{m}(t)$ (and thereby $C$ ) such that $E_{G}=0$, thereby the control algorithm tracks a desired $C$ value neglecting slow and small variations in $V_{\pi m}$. Another benefit of the control is that the frequency dependent AAF and amplified photodiode frequency is automatically equalized, leading for a further improvement in the SNR. When $E_{G}=0$ (17) becomes

$$
Y_{v}(t)=\dot{\theta}(t)
$$

By applying a highpass filter to (19), the low varying phase shift caused by ambient fluctuations can be eliminated, and then integrating $Y_{v}(t)$ gives the required measurement $V_{s}(t)$.

\section{EXPERIMENTAL RESULTS}

The OPM and HVS $V_{\pi}$ was obtained using the Signal Coincidence Method (SCM) measurement technique described in ISO 16063-41 [18], [19]. The respective measured values of $V_{\pi m}=62.3 \mathrm{~V}$ and $V_{\pi s}=4.03 \mathrm{kV}$ are within a $7 \%$ of the theoretical values and were used as system parameters.

The system was designed to have the feedback control loop enabled around $5 \mathrm{kV}( \pm 20 \%)$, i.e. within the necessary condition $V_{s}(t)>V_{\pi s}$. Typical $Y_{1}(t)$ and $Y_{2}(t)$ signals that occur when $E_{G}=0$ are shown in Fig. 5.

When $E_{G}=0, Y_{1}(t)$ and $Y_{2}(t)$ have a $90^{\circ}$ relative phase and equal peak values, i.e. quadrature signals. This condition,

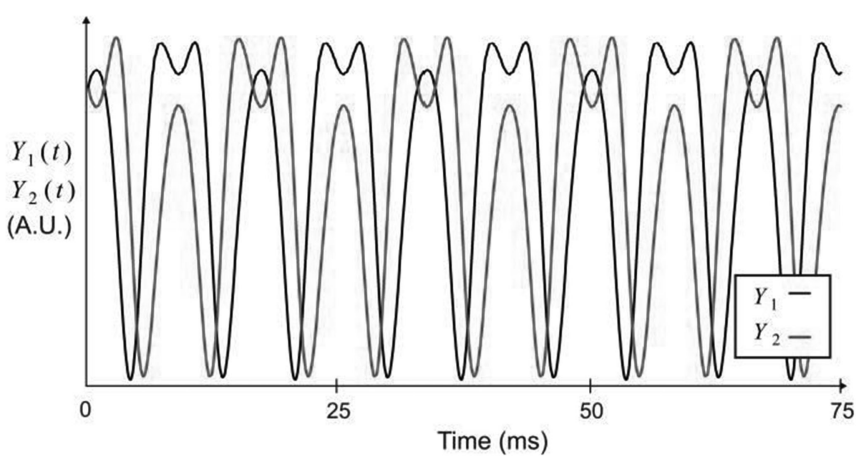

Fig. 5. Typical $Y_{1}(t)$ and $Y_{2}(t)$ signals.

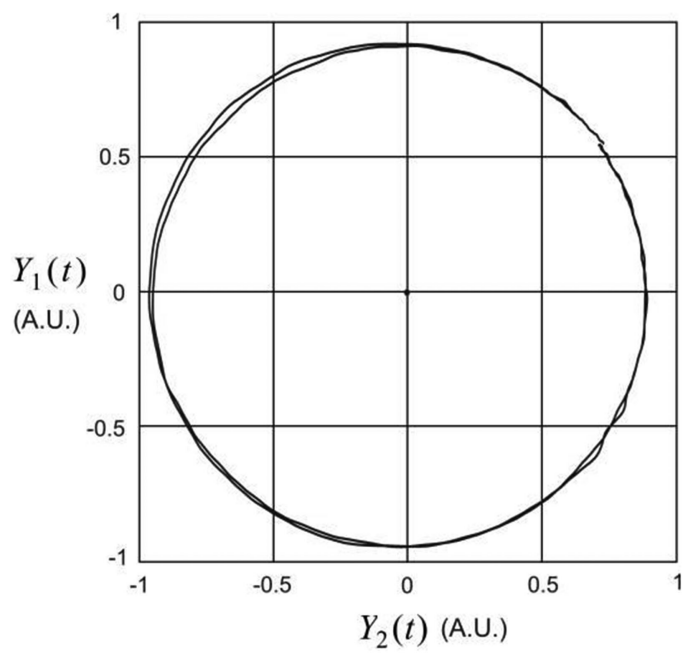

Fig. 6. Typical $Y_{1}(t)$ and $Y_{2}(t)$ quadrature signals.

as observed, is shown in Fig. 6. Although the results can be improved by applying post processing quadrature correction methods [20], the aim of the measurements is show the performance of the system without post processing corrections.

The application is primarily for measurement of power line harmonic distortion. The effective $V_{s}(t)$ applied to the HVS was measured by a calibrated HV probe (Tektronix P6015A $28 \mathrm{kV}) 75 \mathrm{MHz}$ standard and compared to the demodulated signal produced by our scheme. A typical measured and demodulated $60 \mathrm{~Hz} 4 \mathrm{kV}$ signal is shown in Fig. 7

The measured frequency response of our scheme (Syn-p) was compared with measurements obtained using SCM [21]. The SCM method measures the phase induced by the HVS $\varphi(t)$ by nulling the phase induced by the OPM $\Phi(t)$, i.e. with no carrier $\left(V_{m}(t)=0\right)$. The SCM and Syn-p frequency response was compared, as shown in Fig. 8. The approximate detection bandwidth is $4 \mathrm{kHz}$, which is possibly due to the non-ideal filters. The demodulation algorithm run time is $9 \mathrm{~ms}$ and the acquisition time is $20 \mathrm{~ms}$.

A measured sinusoidal voltage, $V_{s}(t)$ (known) at frequency $60 \mathrm{~Hz}$ was applied to the HVS leading to an unknown induced phase $\varphi(t)$. The acquired $v(t)$, related to $\varphi(t)$ in (1), was processed using the Syn-p method to obtain a demodulated $V_{s}(t)$. 


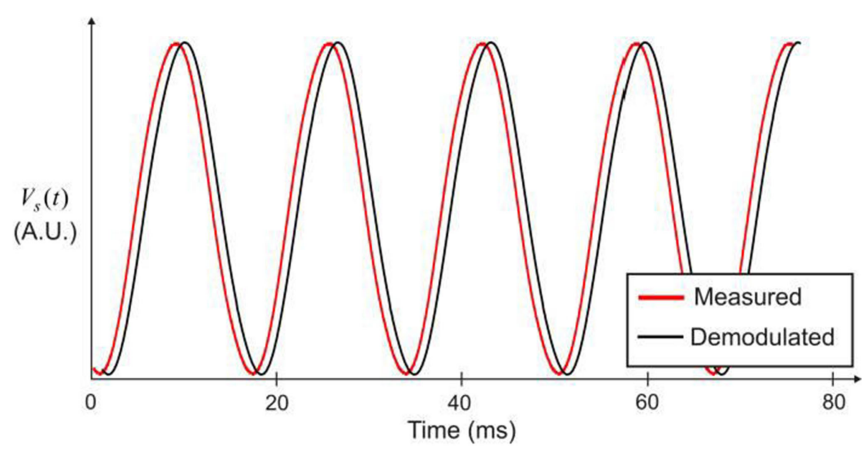

Fig. 7. Measured and time shifted demodulated $V_{s}(t)$.

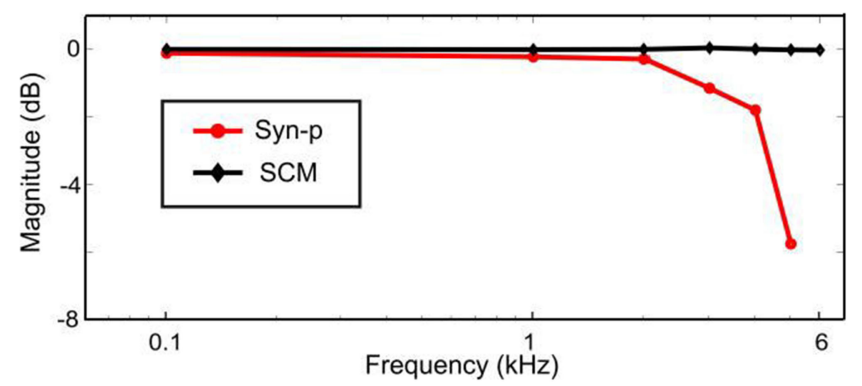

Fig. 8. HVS frequency response obtained using SCM and Syn-p.

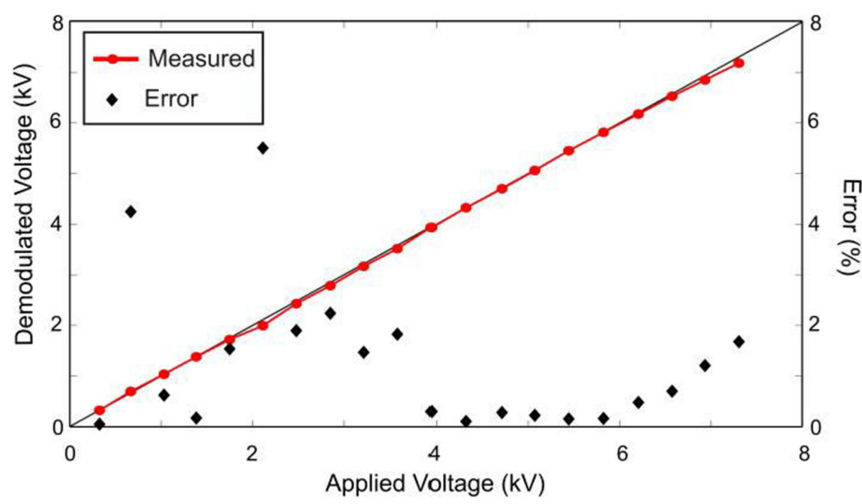

Fig. 9. Comparison between applied and demodulated voltage end relative error.

The comparison, shown in Fig. 9, shows good linearity over a $300-7500 \mathrm{~V}$ range of drive voltage amplitude.

The relative error between the applied and demodulated $V_{s}(t)$ (Fig. 9) was less than $0.3 \%$ over a $4-6 \mathrm{kV}$ range, in which the dynamic control is acting. When $V_{s}(t)<V_{\pi s}(4.03 \mathrm{kV})$ the feedback loop is disabled and the demodulation scheme uses the last stored value of $A$ and $C$, which leads to an increase in the measurement error. The error may be reduced if instead using stored $A$ and $C$ values, the controller switches to a value of $C>\pi$ located near the second maximum value of $\left|J_{0}(C) J_{1}(C)\right|$ (0.0886); e.g. if $C=\pi$ then $\left|J_{0}(C) J_{1}(C)\right|=0.0863$ (Fig. 3), but this leads to a decrease in the SNR. When $V_{s}(t)>6 \mathrm{kV}$, the number of significant photodetected signal harmonics increases. Because of the non-ideal filter design some of the

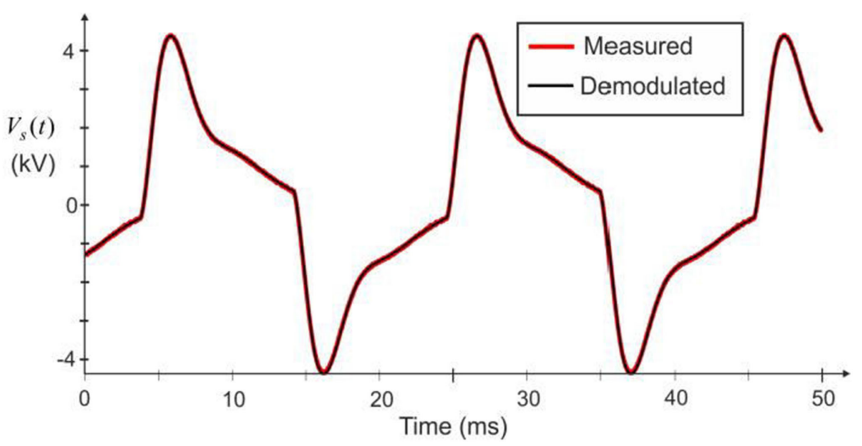

Fig. 10. Frequency method response.

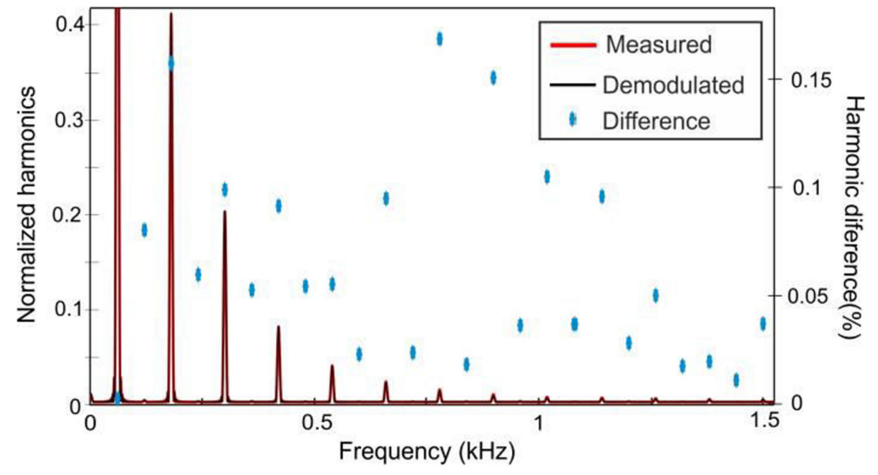

Fig. 11. Measured applied signal and demodulated signal in the frequency domain.

upper-sideband voltage signal induced harmonics present in $S_{1}$ overlap with the lower voltage signal induced harmonics present in $S_{2}$ and vice versa. The error can be reduced by increasing the value of $f_{0}$ at the expense of the required higher speed signal acquisition. As stated above a relative error of $+/-20 \%$ is acceptable [17].

The Total power line Harmonic Distortion (THD) is evaluated according to [22] as

$$
T H D=\frac{\sqrt{\sum_{2}^{25} h_{i}^{2}}}{h_{1}}
$$

where $h_{i}$ is the $i$-th harmonic of the measured signal. In order to verify the proposed method capability to measure THD a highly distorted $4 \mathrm{kV}$ signal was applied to the HVS. The measured (applied) and the demodulated signals are shown in Fig. 10. The $h_{i}$ harmonic was measured by calculating the fast Fourier transform of the acquired signal from which a $T H D_{m}=47.92$ was determined. The total distortion calculated from the demodulated signal was $T H D_{d}=48.09$, i.e. a relative error of $0.35 \%$. The harmonics were normalized by the first harmonic magnitude $(60 \mathrm{~Hz})$ and the differences, between the applied (measured) and demodulated signals are shown in Fig. 11 along with the harmonics.

Temperature variations can affect the detected laser power and the interferometer visibility; however the method is selfcalibrated and independent of ambient fluctuations that affect the product $A V$. The orientation of the OPM and HVS optical 
axes are different so temperature drifts affect $V_{\pi m}$ and $V_{\pi s}$ unequally. The OPM and HVS were maintained stress free in order to minimize the temperature dependence of the crystal dimensional ratio $Y / Z$. The temperature dependence of $r_{22}$ is much less than $r_{33}$ and $r_{13}$ so the temperature sensitivity of $V_{\pi s}$ is substantially larger than that of $V_{\pi m}$ [23]. The temperature dependence of $V_{\pi s}$ is not taken into account; however there are crystal configurations which have almost no temperature dependence [24].

\section{CONCLUSIONS}

A new synthetic-heterodyne demodulation scheme was used for the measurement of HV signal. The use of a control loop results in significant improvements in the detected signal stability and accuracy. The system was successfully used to measure HV signals with high harmonic contents. The experimental results show good agreement with measurements taken using the industry standard SCM technique and consequently the new scheme has many potential applications for HV sensing.

\section{REFERENCES}

[1] S. Kucuksari and G. G. Karady, "Experimental comparison of conventional and optical VTs, and circuit model for optical VT," IEEE Trans. Power Del., vol. 26, no. 3, pp. 1571-1578, Jul. 2011.

[2] W. Chu, S. Kim, X. Wu, L. Wen, and M. Oh, "Optical voltage sensors based on integrated optical polarization-rotated reflection interferometry," J. Lightw. Technol., vol. 34, no. 9, pp. 2170-2174, May 2016.

[3] X. Feng et al., "Birefringence elimination of bismuth germanate crystal in quasi-reciprocal reflective optical voltage sensor," Appl. Opt., vol. 52, no. 8, pp. 1676-1681, Mar. 2013.

[4] W. Chu, S. Heo, and M. Oh, "Polymeric Integrated-optic bias chip for optical voltage transducers," J. Lightw. Technol., vol. 32, no. 24, pp. 4128-4131, Dec. 2014

[5] C. Li and R. Zeng, "Optical voltage sensor using single fresnel rhomb $\mathrm{Bi}_{4} \mathrm{Ge}_{3} \mathrm{O}_{12}$ crystal," IEEE Sensors J., vol. 14, no. 1, pp. 79-84, Jan. 2014

[6] F. Pan, X. Xiao, Y. Xu, and S. Ren, "Optical AC voltage sensor based on two $\mathrm{Bi}_{4} \mathrm{Ge}_{3} \mathrm{O}_{12}$ crystals," IEEE Trans. Instrum. Meas., vol. 61, no. 4, pp. 1125-1129, Apr. 2012

[7] K. Bohnert, S. Wildermuth, A. Frank, and H. Brändle, "Fiber-optic voltage sensor using fiber gyro technology," Procedia Eng., vol. 5, pp. 1091-1094, Sep. 2010

[8] B. Sarkar, C. Koley, N. K. Roy, and P. Kumbhakar, "Condition monitoring of high-voltage transformers using fiber Bragg grating sensor," Measurement, vol. 74, pp. 255-267, Oct. 2015.

[9] B. de Assumpcao Ribeiro, M. M. Werneck, and J. L. da Silva-Neto, "Novel optimization algorithm to demodulate a PZT-FBG sensor in AC high voltage measurements," IEEE Sensors J., vol. 13, no. 4, pp. 1259-1264, Apr. 2013

[10] X. Chen et al., "Optical fiber voltage sensor based on michelson interferometer using phase generated carrier demodulation algorithm," IEEE Sensors J.l, vol. 16, no. 2, pp. 349-354, Jan. 2016.

[11] A. Yariv and P. Yeh, Waves in Crystals: Propagation and Control of Laser Radiation. New York, NY, USA: Wiley, 1984

[12] J. Galeti, C. Kitano, and M. Connelly, "Improved synthetic-heterodyne Michelson interferometer vibrometer using phase and gain control feedback," Appl. Opt., vol. 54, no. 35, pp. 10418-10424, Dec. 2015.

[13] B. Picinbono, "On instantaneous amplitude and phase of signals," IEEE Trans. Signal Process., vol. 45, no. 3, pp. 552-560, Mar. 1997.

[14] M. Feldman, "Hilbert transform in vibration analysis," Mech. Syst. Signal Process., vol. 25, no. 3, pp. 735-802, Apr. 2011.

[15] J. H. Galeti, R. T. Higuti, E. C. N. Silva, and C. Kitano, "Nanodisplacement measurements of piezoelectric flextensional actuators using a new interferometry homodyne method," IEEE Trans. Instrum. Meas., vol. 64, no. 5, pp. 1256-1265, May, 2015.
[16] M. J. Connelly, J. H. Galeti, and C. Kitano, "Michelson interferometer vibrometer using self-correcting synthetic-heterodyne demodulation," Appl. Opt., vol. 54, no. 18, pp. 5734-5738, Jun. 2015.

[17] Instrument Transformers-Part 3: Additional Requirements, for Inductive Voltage Transformers, IEC 61869-3:2011, 2011.

[18] Methods for the Calibration of Vibration and Shock Transducers Part 41 : Calibration of Laser Vibrometers (International Organization for Standardization (ISO)), International Standard ISO16063-41, 2011.

[19] Methods for the Calibration of Vibration and Shock Transducers-Part 11: Primary Vibration Calibration by Laser Interferometry" ((ISO, 1999, reviewed and confirmed), International Standard ISO 16063-11, 2009.

[20] L. Watkins and M. Collett, "Ellipse fitting for interferometry. Part 2: Experimental realization," Appl. Opt., vol. 53, no. 32, pp. 7697-7703, Nov. 2014.

[21] V. Martens and Hans-Jürgen, "Invited article: Expanded and improved traceability of vibration measurements by laser interferometry," Rev. Sci. Instrum., vol. 84, 2013, Art. no. 121601.

[22] Instrument Transformers--The Use of Instrument Transformers for Power Quality Measurements, IEC/TR 61869-103, 2012

[23] J. D. Zook, D. Chen, and C. N. Otto, "Temperature dependence and model of the electro-optic effect in $\mathrm{LiNbO}_{3}, "$ Appl. Phys. Lett., vol. 11, no. 5, pp. 159-161, 1967.

[24] W. Sima, T. Liu, Q. Yang, R. Han, and S. Sun, "Temperature characteristics of Pockels electro-optic voltage sensor with double crystal compensation," AIP Adv, vol. 6, no. 5, pp. 055109-1-05510-10, 2016.

Jose Henrique Galeti (S'12) received the B.Sc. degree in electrical engineering from the Escola de Engenharia Mauá, São Paulo, Brazil, in 1988, and the M.Sc. degree in electrical engineering from Universidade Estadual Paulista, São Paulo, Brazil, in 2012, where he is currently working toward the Ph.D. degree. He was with Hewlett-Packard Brazil, São Paulo, Brazil, and from 1988 to 2009, he was with ETS-Electronic Test Systems, São Paulo, Brazil, where he was involved in test and measurement industrial applications. His current research interests include interferometry, automation, nanodisplacement measurements, piezoelectric actuators, and optical high-voltage sensors.

Ricardo Tokio Higuti (M'00) received the B.S. degree in electrical engineering and the M.S. and Dr.Eng. degrees in mechanical engineering from the Escola Politécnica da Universidade de São Paul, São Paulo, Brazil, in 1991, 1994, and 2001, respectively. Since 1995, he has been with the Department of Electrical Engineering, Universidade Estadual Paulista, São Paulo. His current research interests include signal processing, the development of ultrasonic sensors for liquids, and nondestructive testing.

Cláudio Kitano received the Diploma degree in electrical engineering from the Universidade Estadual Paulista (UNESP), São Paulo, Brazil, the Master's degree in electronics engineering from the Technological Institute of Aeronautics (ITA), São José dos Campos, Brazil, and the D.Sc. degree in microwave and optoelectronics from ITA, in 2001. Since 1987, he has been with the Department of Electrical Engineering, UNESP. His current research interests include optical interferometry, optical fiber sensors, integrated optics, photothermal science, elastic guided waves, acoustic optic, and optical high-voltage sensors.

Michael J. Connelly (S'89-M'92-SM'14) received the B.E. and Ph.D. degrees in electronic engineering from the National University of Ireland, Dublin, Ireland, in 1987 and 1992, respectively. He is an Associate Professor in electronic engineering and the Director of the Optical Communications Research Group, University of Limerick, Limerick, Ireland. His current research interests include coherent optical communication systems, semiconductor optical amplifiers, optical coherence tomography, and optical interferometry. 